Diabetologia 10, 19-22 (1974)

(C) by Springer-Verlag 1974

\title{
Pharmacological Fibrinolysis in Diabetes Mellitus
}

\author{
R. Chakrabarti and G.R. Fearnley \\ Gloucester Royal Hospital, Gloucester, England \\ Received: March 5, 1973, and in revised form: August 13, 1973
}

\begin{abstract}
Summary. The fibrinolytic activity, plasma fibrinogen and blood sugar levels of 20 maturity-onset diabetics were measured before treatment with a) phenformin and b) phenformin plus ethylestrenol; subsequently fortnightly estimations of these parameters were made. After a period of stabilisation, treatments were interchanged between the two groups. It has been shown that phenformin alone only partially increases fibrinolytic activity; the maximum effect was obtained with the combined therapy of phenformin plus ethylestrenol. This treatment also effected a reduction of nearly $40 \%$ in the
\end{abstract}

plasma fibrinogen level from pre-treatment levels. If enhanced fibrinolytic activity in diabetic patients is to be achieved, the results indicate that combined therapy is essentially more effective than therapy with phenformin alone. Blood sugar levels were adequately controlled, as expected, with diet and phenformin alone, addition of ethylestrenol making no difference.

Key words: Fibrinolysis, fibrinogen, maturity-onset diabetes, phenformin and ethylestrenol.
The prevalence of vascular disease amongst diabetics is higher than that amongst a) other disease groups (e.g. autopsy studies [1]), and b) the general population [2]. Various factors have been reported responsible for this difference. It has been shown that blood under normal physiological conditions possesses fibrinolytic activity [3] and a concept has been advanced of a continuous dynamic equilibrium between coagulation and fibrinolysis at sites of injury to the vascular endothelium [4], according to which defective fibrinolysis predisposes to susceptibility to thrombotic episodes. We earlier showed that diabetics as a group tend to have lower levels of fibrinolytic activity than non-diabetics [5]. The results of different studies on this problem have been complicated by the fact that most drugs affecting glycaemia also alter fibrinolytic activity (e.g. insulin [6], sulphonylureas [7], and biguanides [8] for variable lengths of time), and so the fibrinolytic status of diabetics on treatment will tend to be obscured by the effect of treatment. After several years of study of the effects of various drugs on fibrinolysis we have been able to show that a combination of phenformin (50 mg cap. b.d.) and ethylestrenol (4 mg b.d.) given orally will a) enhance blood fibrinolytic activity, b) lower plasma fibrinogen, c) lower serum cholesterol and will maintain this state for a minimum period of 3 years [9]. The present study has been designed to evaluate the effect of these drugs in maturity-onset diabetics.

\section{Materials and Methods}

The subjects were referred to the out-patient department for the diagnosis and treatment of suspected diabetes mellitus. All blood samples were taken between 10 and 11 a.m. in out-patients; the first samples were taken on the day the patients were having their diagnostic glucose tolerance tests, i.e. one hour after the ingestion of $50 \mathrm{~g}$ glucose in the fasting state. Patients were randomly allocated to two groups for different treatment regimes. All patients were reviewed and blood tests repeated at two weekly intervals.

There were 10 patients in each group. 11 were males and 9 females, the distribution in Groups $A$ and $B$ being 5 and 5 , and 6 and 4 , respectively. Their ages ranged from 42 to 79 , the mean age in Group $A$ being 58 and in Group B 61 .

The patients on the first regime (Group A) were treated with phenformin cap. $50 \mathrm{mg}$ b.d. and ethylestrenol $4 \mathrm{mg}$ b.d. for 10 weeks, after which ethylestrenol was stopped, and measurements were repeated on two further occasions while phenformin only was being administered (Fig. 1). Patients on the second regime (Group B) were put on phenformin cap. $50 \mathrm{mg}$ b.d. for 12 weeks, after which ethylestrenol was added and patients were followed for a further 6 weeks. At this stage ethylestrenol was stopped, but phenformin was continued for a further 2 weeks (Fig. 2). All patients were seen initially, after the glucose tolerance test, by the dietician and appropriate restricted carbohydrate diets, dictated by level of glycaemia, body weight and age were advised. Blood fibrinolytic activity and fibrinogen were measured by the method described by Fearnley et al. [9]. Blood sugar was measured by the Folin and $\mathrm{Wu}$ method.

Obesity was estimated by the index: (Weight/ height) 1.7 [10].

\section{Results \\ 1. Fibrinolytic Activity}

a) Relation to Obesity Index and Blood Sugar Levels. Blood fibrinolytic activity is inversely related to lysis 
time. Table 1 shows the age, initial lysis times, fibrinolytic activity, obesity index, and blood sugar levels of the 20 subjects. Only the correlation coefficient between the obesity index and blood sugar levels was significant $(P<0.001)$.

Table 1. Shows age, initial lysis time, fibrinolytic activity (reciprocal of lysis time), obesity index and blood glucose levels of 20 maturity-onset diabetics

\begin{tabular}{lclll}
\hline Age & Lysis time & $\begin{array}{l}\text { Fibrinolytic } \\
\text { activity }\end{array}$ & $\begin{array}{l}\text { Obesity } \\
\text { index }\end{array}$ & Glucose \\
\hline 66 & 5.5 & 0.182 & 0.134 & 264 \\
42 & 24 & 0.042 & 0.118 & 210 \\
63 & 9 & 0.111 & 0.115 & 168 \\
57 & 21.75 & 0.046 & 0.126 & 210 \\
79 & 6 & 0.167 & 0.102 & 294 \\
50 & 7 & 0.143 & 0.155 & 452 \\
64 & 20.5 & 0.049 & 0.154 & 488 \\
68 & 20 & 0.050 & 0.153 & 400 \\
63 & 24 & 0.042 & 0.127 & 444 \\
59 & 13 & 0.077 & 0.127 & 360 \\
47 & 21.25 & 0.047 & 0.153 & 360 \\
63 & 19.5 & 0.051 & 0.137 & 295 \\
52 & 16 & 0.062 & 0.117 & 264 \\
48 & 8 & 0.125 & 0.109 & 220 \\
58 & 11 & 0.091 & 0.136 & 384 \\
56 & 24 & 0.042 & 0.133 & 262 \\
64 & 6 & 0.167 & 0.114 & 224 \\
62 & 8 & 0.125 & 0.121 & 308 \\
62 & 11 & 0.091 & 0.118 & 312 \\
68 & 18 & 0.056 & 0.114 & 382 \\
\hline
\end{tabular}

Correlations between: Fibrinolytic activity and obesity : 0.3805 (not significant). Fibrinolytic activity and glucose: 0.2405 (not significant). Obesity and glucose: $0.7116(P<0.001)$.

b) Effect of Treatment. (One patient in Group B had a significant increase of fibrinolytic activity maintained at 12 weeks, so ethylestrenol was not added to his treatment. This result is excluded in subsequent calculations. Fig. 1 shows the changes in mean fibrinolytic activity of the 10 patients in Group A. It is evident that treatment with phenformin and ethylestrenol together promptly increases fibrinolytic activity, but that when ethylestrenol is stopped, fibrinolytic activity reverts within 4 weeks almost to pre-treatment levels. The effect of phenformin alone, comparing results in week 0 and results in week 16, was unremarkable. The results of treatment in Group B are shown in Fig. 2, which shows that phenformin alone only partially increases fibrinolytic activity, but that as soon as the combined therapy is started, there is a significant increase of fibrinolytic activity. That this increase is due to the combination of drugs is confirmed when ethylestrenol is stopped and fibrinolytio activity reverts to the level achieved with phenformin alone. (The effect of ethylestrenol on its own was not studied as it has been shown that its effect is temporary [8] and moreover we could not justifiably treat a group of diabetics with ethylestrenol alone). For fibrinolytic activity phenformin alone is not significantly better than no treatment $(0.05>P<0.1)$.

\section{Blood Sugar}

As expected, the blood sugar was well controlled by diet and phenformin. The addition of ethylestrenol to the treatment did not make any difference to blood sugar levels.

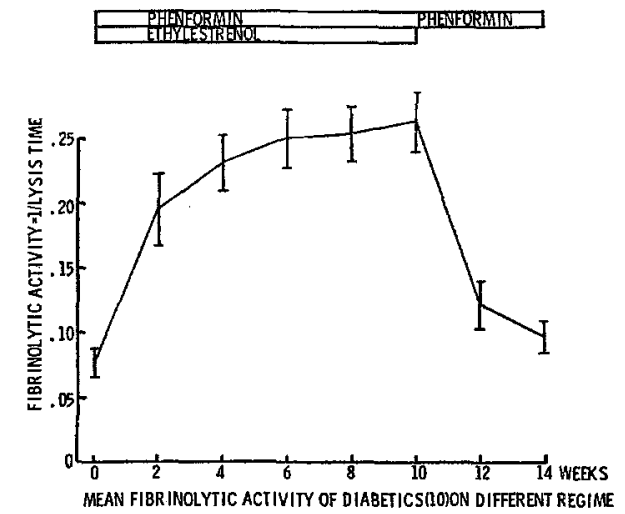

Fig. 1. Shows mean fibrinolytic activity of 10 subjects with \pm standard error of mean in Group A during the course of treatment

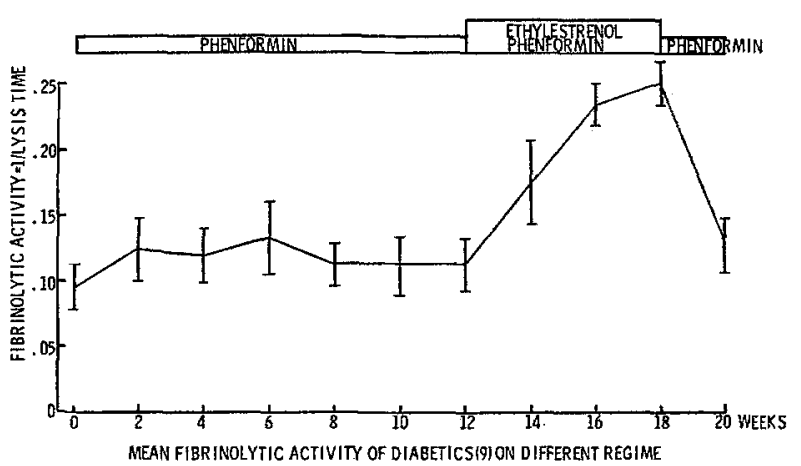

Fig. 2. Shows mean fibrinolytic activity of 9 subjects with \pm standard error of mean in Group $B$ during the course of treatment

\section{Plasma Fibrinogen}

Fig. 3 shows the mean plasma fibrinogen levels of the 10 subjects in Group A. There is a reduction of about $30 \%$ in the mean fibrinogen level with treatment with phenformin and ethylestrenol and there is a slight rise (about 10\%) when ethylestrenol is withdrawn. Fig. 4 shows the mean fibrinogen levels in Group B. With phenformin alone the mean fall in fibrinogen level is about $25 \%$, but when ethylestrenol is added the reduction is about $38 \%$. Both treatment regimes lowered fibrinogen significantly $(P<0.01)$.

\section{Discussion}

A number of factors contributing to the greater incidence of cardiovascular disease in diabetics has been suggested, e.g. micro-angiopathy [11], hyperglycaemia [12], hypertension [13], and hyperlipidaemia [14]. Some of the features that distinguish diabetic pa- 
tients with ischaemic heart disease from non-diabetics with ischaemic heart disease are a) increase in heart disease incidence in diabetic women who are free from recognised risk factors, namely normotensive, premenopausal, and free of significant hyperlipidaemia b) a higher mortality rate and shorter life expectancy amongst survivors of myocardial infarction amongst diabetics [15]. These considerations led us to investigate the blood fibrinolytic activity of diabetic males and females [5], and we showed that diabetics as a group have lower fibrinolytic activity than agematched non-diabetics; there was a significant negative correlation between obesity and fibrinolytic activity, which has been confirmed by others in non-diabetics [16]. In the present study, the number is rather small and the absence of a significant correlation be-
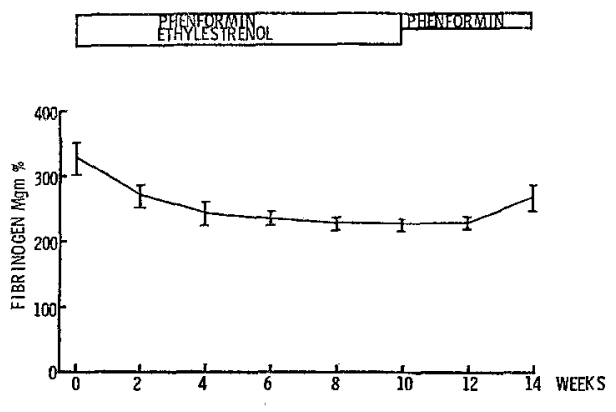

MEAN FIBR INOGEN LEVELS OF DIABETICSHO) ON DIFFERENT REGIME

Fig. 3. Shows mean plasma fibrinogen levels with \pm standard error of mean in 10 subjects in Group A during the course of treatment
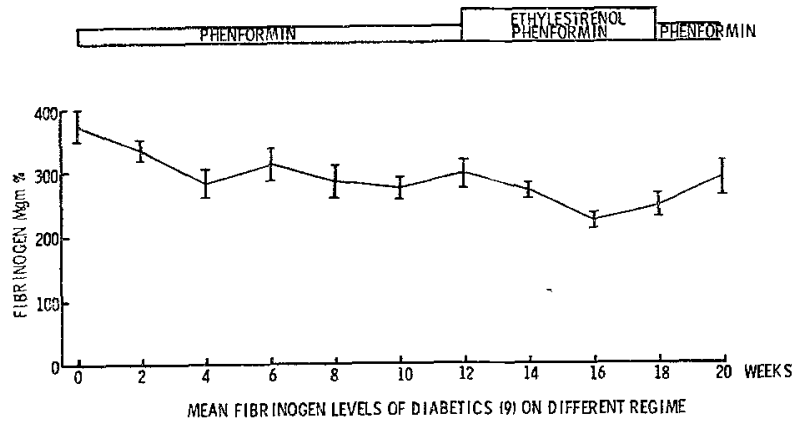

Fig. 4. Shows mean plasma fibrinogen levels with \pm standard error of mean in 9 subjects in Group B during the course of treatment

tween obesity and fibrinolytic activity is possibly due to the very high proportion of subjects with low fibrinolytic activity amongst these patients with maturity-onset diabetes. The relative roles of coagulation factors, fibrinolysis and platelets in vascular disease, particularly in diabetics, are undetermined, but abnormalities in some of these factors have been documented. Hence it may be important to explore and establish the effect of treatment on some of these parameters. A number of drugs have been shown to enhance fibrinolysis but this effect is short-lived. Re- sistance to drugs appears quickly and necessitates increasing doses [8]. In a long term follow-up of arteriosclerotic subjects it has been shown that a combination of phenformin and ethylestrenol in the dosage used in this study had the best results. Resistance to phenformin alone appears in a large percentage of cases in 3-6 months [9]. It has recently been shown that it is feasible to treat a group of patients with ischaemic heart disease with phenformin and ethylestrenol in a dosage used in this study for a period of 3 years and to maintain an enhanced fibrinolytic state [17]. This study suggests an equivalent effect of the drugs on fibrinolysis and on plasma fibrinogen in diabetics. It also emphasises that treatment with phenformin alone has only a slight effect on these parameters, particularly fibrinolytic activity, compared with that of phenformin and ethylestrenol.

It has also been shown that this combination of drugs decreases platelet adhesiveness to glass in arteriosclerotic patients [18], and work is in progress to see if this applies to diabetics, since it has been shown that platelet adhesiveness to glass is increased in diabetics [19].

Acknowledgements. We acknowledge with thanks the technical help of Mr. J.F. Evans and Miss E. Cronow. We also thank Dr. A. Nicol, Consultant Pathologist at Gloucester for allowing some of his patients to take part, the patients for their cooperation, and Mr. W.R.S. North, Statistician to the Epidemiology and Medical Care Unit at Northwick Park, for help with statistical analysis of the results.

\section{References}

1. Bell, E.T.: A post-mortem study of vascular disease in diabetics. Arch. Path. 53, 444-455 (1952)

2. Keen, H., Rose, G., Pyke, D.A., Boyns, D., Chlouvratis, C., Mistry, S.: Blood sugar and arterial disease. Lancet $1965 \mathrm{II}, 505-508$

3. Fearnley, G.R., Tweed, J.M.: Evidence of an active fibrinolytic enzyme in the plasma of normal people with observations on inhibitions associated with the presence of calcium. Clin. Sci. 12, 81-89 (1953)

4. Astrup, T.: The biological significance of fibrinolysis. Lancet $1965 \mathrm{II}, 565-567$

5. Fearnley, G.R., Chakrabarti, R., Avis, P.R.D.: Blood fibrinolytic activity in diabetes mellitus and its bearing on ischaemic heart disease in obesity. Brit, med. J. 1963 I, $921-923$

6. Fearnley, G.R., Vincent, C.T., Chakrabarti, R.: Reduction of blood fibrinolytic activity in diabetes mellitus by insulin. Lancet 1959 II, 1067

7. Fearnley, G.R., Chakrabarti, R., Vincent, C.T.: Effect of the sulphonylureas on fibrinolysis. Lancet $1960 \mathrm{II}$, $622-625$

8. Fearnley, G.R., Chakrabarti, R.: Pharmacological enhancement of fibrinolytic activity of blood. J. clin. Path. 17, 328-332 (1964)

9. Fearnley, G.R., Chakrabarti, R., Hocking, E.D.: Fibrinolytic effects of biguanides plus ethylestrenol in occlusive vascular disease. Lancet 1967 II, 1008-1011

10. Newens, M.E., Goldstein, H. : Height, weight and the assessment of obesity in children. Brit. J. prev. soc. Med. 26, 33-39 (1972) 
11. Havel, R.J.: Arteriosclerosis and diabetes in small blood vessel involvement in Diabetes Mellitus, p. 114 (Ed. Siperstein, M.D.). Washington D.C.: Amer. Inst. biol. Sci. 1964

12. Cleave, T.L., Campbell, G.D.: Diabetes, coronary thrombosis and the saccharine disease, p. 106. Bristol: John Wright \& Sons Ltd.

13. Wetherby, M.: A comparison of blood pressure in men and women. Ann. int. Med. 6, 754-760 (1932)

14. Mann, G.V.: Metabolism of serum lipids in diabetes and in arteriosclerosis. Diabetes $4,273-279$ (1955)

15. Bradley, R.F., Partamian, J.O.: Coronary heart disease in the diabetic patient. Med. Clin. N. Amer. 49, 1093-1104 (1965)

16. Ogston, D., McAndrew, G.M. : Fibrinolysis in obesity. Lancet 1964 II, 1205-1207

17. Chakrabarti, R., Fearnley, G.R.: Phenformin and ethylestrenol in survivors of myocardial infarction. Lancet 1972 II, $556-559$
18. Chakrabarti, R., Fearnley, G.R. : Reduction of platelet stickiness by phenformin plus ethylestrenol. Lancet 1967 II, 1012 - 1014

19. Mayne, E.E., Bridges, J.M., Weaver, J.A.: Platelet adhesiveness, plasma fibrinogen and factor VIII levels in diabetes mellitus. Diabetologia 6, 436-440 (1970)

Dr. R. Chakrabarti MRC/DHSS Epidemiology and Medical Care Unit Northwick Park Hospital

Watford Road,

Harrow

Middlesex HA 13 UJ

England 